\title{
Immunoreactivity of the Body During the Surgical Treatment of Brain Tumors
}

\author{
Mitalip Mamytov \\ Department of Neurosurgery, Kyrgyz State Medical Academy, Kyrgyzstan, Bishkek
}

Email address:

mitalipmamytov@gmail.com

\section{To cite this article:}

Mitalip Mamytov. Immunoreactivity of the Body During the Surgical Treatment of Brain Tumors. Clinical Neurology and Neuroscience. Vol. 4, No. 4, 2020, pp. 71-75. doi: 10.11648/j.cnn.20200404.11

Received: September 26, 2020; Accepted: October 13, 2020; Published: October 21, 2020

\begin{abstract}
Background: The cells of the immune system are actively involved in the tumor development process and can either suppress or stimulate tumor growth. Objective: to determine the role and significance of immunological indications of patients with brain tumors of different histostructure before and after surgical treatment. Methods: The number of lymphocytes, neutrophilic granulocytes, and platelets was analyzed in 246 patients with brain tumors of various histostructures. Results: It was determined that there were significant fluctuations in the content of leukocytes in the blood of patients with brain tumors of various histogenesis. The largest number of leukocytes was observed in neuroectodermal and mesenchymal tumors. However, neuroepithelial tumors (pituitary adenomas) did not show a considerable increase in the number of leukocytes compared with that in healthy individuals. Conclusions: It is revealed that the determination of the immune response is an essential component of clinical examination. These immune response options allow to develop targeted immunotherapy. The determination of immunoreactivity for these options was determined to be clinically feasible. In addition, belonging to a particular group based on the immune status considerably determines the further course of the tumor process and the effectiveness of the surgical treatment of brain tumors.
\end{abstract}

Keywords: Brain, Immunology, T, B Lymphocytes, Immunoglobulins, Brain Tumors, Surgical Treatment (Outcomes and Effectiveness of the Operation)

\section{Introduction}

The cells of the immune system are actively involved in the tumor development process and can either suppress or stimulate tumor growth [1]. During the last decade, considerable research efforts have been made to study the role of innate immunity cells (e.g., neutrophilic granulocyte, natural killer cells, and dendritic cells in tumorigenesis) [2, 3] At the same time, considerable attention has been paid to platelets, which are classified as immunoregulatory cells and are involved in the processes of hemocoagulation of thrombosis, adhesion of pathogens, suppression of the function of immune cells, and stimulation of tumor growth [4].

The molecular cellular mechanisms of the interaction of platelets and neutrophilic granulocytes both with tumor cells and with macrophages and lymphocytes has been previously described [4]. For the practical use the data obtained, it is recommended to determine both the absolute number of leukocytes, lymphocytes, neutrophilic granulocytes, and their ratio in the blood of cancer patients. The absolute and relative content of innate and acquired immunity cells in peripheral blood is already used to predict the duration of the remission period of some oncological diseases, in particular in cancer processes and brain tumors $[5,6]$. In fact, there are many problems in the field of neuroimmunology, but in a journal article, it is very difficult to list all the existing problems, so we limited ourselves to indicating the most relevant aspects of humoral and cellular immunology in brain tumors).

In glioblastomas with more than $4 \mathrm{ng} / \mathrm{lph}$ ratio, the duration of postoperative remission exceeds 11 months [7]. At the same time, the blood content of innate and acquired immunity cells for many tumors, including brain tumors of various origins and degrees of anaplasia, has not been studied enough, and the prognostic value of the presented indicators is controversial.

The goal of this study was to determine the role and importance of immunological parameters in assessing the 
clinical and morphological features of brain tumors and the effectiveness of their surgical treatment, as well as to study the content of immune cells in peripheral blood in patients with brain tumors of different histostructures.

In recent years, new data on the biology of malignant gliomas have been obtained, a number of antigenic characteristics of gliomas and their dependence on genetic qualities have been established. The state within tumor and systemic immune responses, as well as their dependence on the degree of tumor malignancy, has been studied in detail [8, 9].

Very intensive and extensive researches are underway to develop various methods of immunotherapy for the treatment of malignant gliomas, at the same time, the study of the heterogeneity of malignant gliomas at the genetic and immunological level remains a challenge of the time [10-12].

\section{Methods}

The analysis covered the number of lymphocytes, neutrophilic granulocytes, and platelets in 246 patients with brain tumors of various histostructures. In addition, the level of the main classes (e.g., A, M, and G) of neutrophil damaging immunoglobulins (MDI) was determined according to Kitaev's modifications. The leukocytes agglomeration reaction (LAR) was determined according to modifications of David et al. An additional objective was to determine anti-brain autoantibodies using the complement consumption reaction according to Morozov's modifications and to determine circulating immune complexes.

According to the international classification (2016), brain tumors are divided into neuroectodermal tumors - glial 134 (gliomas, astrocytoma, glioblastomas) mesenchymal (meningiomas - 59), ectodermal (pituitary adenomas (craniopharyngioma, germinoma) - 31, others - 22).

A total of $172(69.9 \%)$ patients with brain tumors underwent surgical treatment. The reaction of the body of the patients with brain tumors was studied in dynamics by monitoring a set of humoral and cellular immune responses: before surgery, on the 2nd-5th day, and one month after the surgery. Research results were subjected to statistical processing using non-parametric criteria, which allowed to perform statistical analysis with relatively small samples. The reliability criterion ( $p$ ) was determined according to the Student table $(p<0.05)$. Immunoglobulins, damages to neutrophils, agglomeration of neutrophils - these research methods are widely used in general oncology (tumors of the lungs, mammary gland, tumors of the stomach, bladder, etc.). The indicated immune reactions in brain tumors were almost never carried out, these reactions are indicators of the body's immunoreactivity, and we were convinced that in brain tumors they change depending on the histostructure and severity of surgery and other reasons. Patients with comorbidities were not included in these studies.

\section{Results and Discussion}

The content of leukocytes, neutrophilic granulocytes, lymphocytes, and platelets in the peripheral blood in patients with brain tumors of different histostructures is shown in Table 1. The table shows that there are significant fluctuations in the content of leukocytes in the blood of patients with brain tumors of various histogenesis. The largest number of leukocytes was observed for neuroectodermal and mesenchymal tumors. However, neuroepithelial tumors (pituitary adenomas) did not show a considerable increase in the number of leukocytes compared with those in healthy individuals. These differences in the leukocyte count in tumors of various geneses can be explained by the intense inflammatory response of the body in the first two types of tumors and by possible tumor sizes, which are much smaller for pituitary adenoma than with meningioma. There are inflammatory reactions of the body in malignant tumors, which can serve as additional factors contributing to a more drastic damage to the body's immune mechanisms. Therefore, our studies have shown that in malignant brain tumors, sharper deviations in the parameters of the body's immunoreactivity were established than in pituitary adenoma and meningiomas.

Table 1. Content of immune cells in patients with brain tumors of various histogenesis.

\begin{tabular}{|c|c|c|c|c|}
\hline \multirow{2}{*}{ Tumor histostructure } & \multicolumn{4}{|c|}{ Value of the indicator $(M \pm m)$} \\
\hline & PLT, $\times 10^{9}$ in $1 \mathrm{~L}$ & Leukocytes, $\times 10^{9}$ in $1 \mathrm{~L}$ & $\mathrm{Lph}, \times 10^{9}$ in $1 \mathrm{~L}$ & $\mathrm{NG}, \times 10^{9}$ in $1 \mathrm{~L}$ \\
\hline Neuroectodermal (n-87) & $236.5 \pm 6.8$ & $8.7 \pm 0.36$ & $2.4 \pm 0.09$ & $5.91 \pm 0.36$ \\
\hline Mesenchymal (n-32) & $248 \pm 12.4$ & $8.9 \pm 1.5$ & $2.9 \pm 0.20$ & $6.09 \pm 0.52$ \\
\hline Ectodermal tumors (n-28) & $229 \pm 9.2$ & $6.4 \pm 0.86$ & $1.95 \pm 0.10$ & $3.92 \pm 0.60$ \\
\hline Control group (n-29) & $213.8 \pm 7.2$ & $5.2 \pm 0.34$ & $1.60 \pm 0.09$ & $3.93 \pm 0.56$ \\
\hline
\end{tabular}

Table 2. Content of immune cells in patients with brain tumors of various histogenesis.

\begin{tabular}{llll}
\hline \multirow{2}{*}{ Tumor histostructure } & \multicolumn{3}{l}{ Value of the indicator, standard unit (M \pm m) } \\
\cline { 2 - 3 } & PLT/Lph & PLT/NG \\
\hline Neuroectodermal tumors & $138.2 \pm 9.2$ & $48.9 \pm 2.1$ \\
Mesenchymal tumors & $121.42 \pm 11.4$ & $54.7 \pm 6.4$ & $2.56 \pm 0.31$ \\
Ectodermal tumors & $126.38 \pm 9.8$ & $73.5 \pm 7.2$ & $2.53 \pm 0.41$ \\
Control group & $138.12 \pm 5.46$ & $65.6 \pm 5.8$ & $2.52 \pm 0.64$ \\
\hline
\end{tabular}

Thus, the research results showed that leukocytosis and neutrophilia were occurring by the presence of a relatively small increase in the level of lymphocytes and platelets for neuroectodermal (in particular with glioblastomas) and 
mesenchymal (meningioma) tumors. For neuroepithelial (pituitary adenoma) tumors, the number of leukocytes, platelets, and neutrophilic granulocytes did not change, while the lymphocyte content increased. Thus, different brain tumors were characterized by different changes in the content of the immune system cells.

By studying the ratio of individual cell types (Table 2), the changes in the ratio of immune cells were identified depending on the histology of brain tumors, which to some extent reflects the activity of the body's immune system. The ratio of neutrophilic granulocytes and lymphocytes was the highest for gliomas and glioblastomas, while for mesenchymal and neuroepithelial neoplasms we observed a slight increase in them compared with those in the control group. Our studies showed that the growth of tumors of neuroectodermal and mesenchymal origin was accompanied by changes both in the number and ratio of cells of innate and acquired immunity, which is consistent with the data on the immunosuppression of T-cell immunity and the inflammatory response in the tumors. Our studies have shown ambiguous changes in the ratio of immune cells depending on the histology of brain tumors, which may, to a certain extent, reflect the activity of the body's immune system. The difficulty in understanding the interaction of a tumor with the immune system is that there is a classical idea that the immune system can control the development of a tumor and is even capable of causing its death. But there is also the opposite opinion (based on data from numerous studies) that can promote and even stimulate tumor growth. Attempts to have an immunostimulating effect on the immune system did not lead to the cessation of tumor growth, but, on the contrary, to the rapid progression of the tumor disease. Therefore, the results of our studies have shown ambiguous changes in immune responses in various tumors (hyper - hyporeactive options of the body's immune status) of the brain.

Previous immunological studies on humoral cell types identified immunity deviation either towards suppression or activation [13, 14]. Our studies have shown that both for malignant and benign tumors, the existence of anti-brain and antineoplastic anti-self antibodies was identified in the blood [15].

For malignant tumors, $76 \%$ of patients had anti-brain and antineoplastic anti-self antibodies and in benign tumors - in $48 \%$ of patients. The antibody titer with antigen from the white matter was $6.7 \pm 1.7$ and that from the benign tumor tissue was $-5.9 \pm 1.3$. The most apparent changes were noticed with the antigen from the gray matter and with antigen from malignant tumor, and antibody titers were corresponding to antibodies $14.9 \pm 2.7$ and $15.1 \pm 2.6$, respectively. Therefore, the rate of development of neurosensitization was almost the same for both to antigens from the white matter of the brain, and to antigens from tumors Whereas the level of antibodies to antigens from gray matter and to antigens of malignant tumors would be significantly high (Table 3 ). In our work, we expected to find out the presence and level of deviation of immunological parameters of patients with brain tumors, which could serve as the basis for targeted immunotherapy [16]. In literature there is information about the interaction and interaction of lymphocytes, immunoglobulins and platelets in the process of autosensitization and a decrease in the body's immunoreactivity in some pathological processes.

Table 3. Rate of detection of autoantibodies in patients with brain tumors.

\begin{tabular}{|c|c|c|c|c|c|c|}
\hline \multirow{2}{*}{ Types of tumors } & \multirow{2}{*}{$\mathbf{n}$} & \multicolumn{2}{|l|}{ Rate, \% } & \multicolumn{3}{|l|}{ Antibody titers $\mathbf{M} \pm \mathbf{m}$} \\
\hline & & Brain antibody & Tumor antibody & With brain antibodies & With tumor antibodies & R. \\
\hline Glial tumors & 21 & 76.2 & 71.4 & $13.2 \pm 4.2$ & $9.3 \pm 2.4$ & $<0.01$ \\
\hline Meningiomas & 13 & 54.2 & 69.2 & $8.9 \pm 3.6$ & $10.4 \pm 3.2$ & $<0.1$ \\
\hline Adenomas & 10 & 40.0 & 60.0 & $7.3 \pm 2.4$ & $8.2 \pm 3.4$ & $>0.05$ \\
\hline Neurinomas & 6 & 35.6 & 50.0 & $6.2 \pm 2.3$ & $9.4 \pm 2.9$ & $<0.05$ \\
\hline Control & 20 & 17.4 & - & $2.3 \pm 0.9$ & $2.14 \pm 0.7$ & - \\
\hline
\end{tabular}

In patients with malignant brain tumors, the antibody titer decreased 2-3 days after the operation, while it increased in those with benign tumors. When the general state of patients worsened during the postoperative period, a drop in the level of autoantibody titer was also observed regardless of the nature of the brain tumor. The low titer of antibodies and its weak dynamics in patients with malignant tumors may indirectly indicate the suppression of the function of immunocompetent cells, which can be considered as the effect of toxicity of metabolites and antigens on immunogenesis [17-19], which is an indicator of a decrease in the reactivity of the body and the level of autoimmunity.

One month after the surgery, anti-brain and anti-tumor antibodies continued to exist in $30-43 \%$ of patients with all types of antigens. The antibody titer noticeably decreased in the blood of patients with benign tumors. Only after the total removal of brain tumors by the end of the month after the surgery, the antibody titer became similar to the indicators of the control group, which was not observed after the partial removal of neoplasms.

A study on T-B blood lymphocytes of patients with benign tumors showed that the level of T-lymphocytes was slightly reduced (36.6 \pm 4.9$)$, and the absolute number was considerably reduced $(721.9 \pm 62.2)$ compared with the control data. However, there was no noticeable deviation in B-lymphocyte indicators compared with that of the standard. The average value of T-ROC (rosette-forming cells) in malignant tumors is significantly lower than that of the control data. At the same time, patients with malignant tumors were found to have increased B-ROC [20, 21].

It is known that there is a separation of tumors according to standard histopathology, but we relied on the conclusion of histological studies of a remote tumor of our patients, but unfortunately, we could not deviate from the classification 
versions existing in the literature.

On the 2nd-5th day after surgery, for the patients with benign tumors, the number of T-lymphocytes remained unchanged, while that of B-lymphocytes increased. For malignant tumors, the content of T-lymphocytes became somewhat higher, and that of B-lymphocytes remained unchanged. At the end of one month after surgery, there was an increase in the content of B-lymphocytes (Table 4). The relative number of B-lymphocytes increased when the absolute number decreased in patients with malignant neoplasms during the postoperative period.

Thus, the table shows that with malignant brain tumors there was a significant violation of the T-cell immunity.

The course of all types of tumors was accompanied by a decrease in the levels of $\mathrm{M}$ and $\mathrm{G}$ immunoglobulins compared with the control data and a significant increase in the A immunoglobulin content, which is consistent with data from other authors of the study [15]. It is an important observation that in patients with malignant brain tumors, the ratio of immunoglobulins to each other is significantly disturbed. Thus, if the IgG/A ratio is 8-9 in healthy people, then the IgG level decrease was observed in patients with benign neoplasms and the $\mathrm{IgG} / \mathrm{A}$ ratio was 5.6-6; however, the same ratio was less than 5 for malignant tumors.

Table 4. Dynamics of T- and B-lymphocytes after the surgical treatment of patients with brain tumors.

\begin{tabular}{|c|c|c|c|c|c|}
\hline \multirow{2}{*}{ Examination time } & \multirow{2}{*}{ n } & \multicolumn{2}{|c|}{ T-lymphocytes } & \multicolumn{2}{|c|}{ B-lymphocytes } \\
\hline & & $\%$ & Absolute number & $\%$ & Absolute number \\
\hline \multicolumn{6}{|l|}{ A) Benign tumors } \\
\hline Before surgery & 43 & $36.6 \pm 4.9$ & $721.9 \pm 64.3$ & $10.8 \pm 2.2$ & $205.1 \pm 29.3$ \\
\hline On the 2 nd -5 th day after the surgery & 32 & $31.8 \pm 4.6$ & $778.8 \pm 69.6$ & $13.7 \pm 2.3$ & $316.6 \pm 25.4$ \\
\hline $\mathrm{P}$ & & $<0.05$ & $<0.05$ & 0.1 & $<0.05$ \\
\hline A month after the surgery & 24 & $42.8 \pm 6.2$ & $880.8 \pm 57.3$ & $12.1 \pm 2.6$ & $253.7 \pm 22.3$ \\
\hline $\mathrm{P}$ & & $<0.05$ & $<0.05$ & - & 0.05 \\
\hline \multicolumn{6}{|l|}{ B) Malignant tumors } \\
\hline Before surgery & 25 & $32.3 \pm 4.9$ & $550.3 \pm 37.4$ & $16.3 \pm 3.7$ & $283.5 \pm 26.7$ \\
\hline On the 2 nd-5th day after the surgery & 19 & $28.6 \pm 7.3$ & $665.2 \pm 51.2$ & $13.6 \pm 3.2$ & $283.3 \pm 36.4$ \\
\hline One month after the surgery & 14 & $17.3 \pm 6.7$ & $711.1 \pm 62.4$ & $19.0 \pm 3.6$ & $186.2 \pm 20.2$ \\
\hline
\end{tabular}

On the 2nd-5th day after the surgical treatment, the content of $\mathrm{M}$ and $\mathrm{G}$ immunoglobulins decreased compared with presurgical indicators, and their levels were 79.2 \pm 12.4 and $856.4 \pm 58.4$, respectively. A considerable increase in all main classes of immunoglobulins was observed one month after the tumor removal. Their content became larger than that before the surgery and even approached the indicators of the control group (Table 5).

Table 5. Content of immunoglobulins in the blood of patients with brain tumors.

\begin{tabular}{|c|c|c|c|c|c|}
\hline \multirow{2}{*}{ Group of examined people } & \multirow{2}{*}{$\mathbf{n}$} & \multicolumn{4}{|c|}{ Immunoglobulin $\mathbf{M} \pm \mathbf{m}$} \\
\hline & & A & $\mathbf{M}$ & $\mathbf{Y}$ & $\mathbf{P}$ \\
\hline Donors & 20 & $168.4 \pm 18.0$ & $122.1 \pm 6.8$ & $1604.1 \pm 30.0$ & $<0.05$ \\
\hline With benign tumors & 34 & $119.1 \pm 12.3$ & $89.2 \pm 2.4$ & $1274.8 \pm 74.7$ & $<0.05$ \\
\hline With malignant tumors & 22 & $263.9 \pm 22.6$ & $67.9 \pm 5.2$ & $779.0 \pm 29.0$ & $<0.01$ \\
\hline
\end{tabular}

After analyzing the data on humoral and cellular immune responses and the degree of autosensitization, we were able to establish" four variants of the body's immunoreactivity in brain tumors.

The first option is the hyperergic state of immunity, which was characterized by high rates of immunological reactions. The detected immunological changes were notable for their considerable reversibility during the postoperative period.

The second option is the hypoergic type of the course of immune reactions, which is characterized by reduced rates of both humoral and cellular immune tests. The reversibility of impaired immune processes in the postoperative period is negligible.

The third option is the anergic type of body immunity towards tumor growth.

This type of immune response is primarily characterized by reduced immunological parameters such as the absence of a skin reaction to tuberculin, low level of antibodies and immunoglobulins in the blood, and low percentage of $\mathrm{T}$ lymphocytes.
The fourth option is the "paradoxical" type, which was encountered in several patients with both benign and malignant tumors. These patients had low indicators of humoral immunity, and the cellular reactions of these patients were at low levels.

\section{Conclusions}

Thus:

1. Our studies have shown that with brain tumors, significant violations of both cellular and humoral immunity are observed. The level of indicators of immune disorders depends on the degree of malignancy of the tumors and the effectiveness of the surgical treatment of brain tumors.

2. We believe that it is essential to determine the immune response as a component of clinical examination.

3. These immune responses allow to develop targeted immunotherapy $[16,22]$.

4. The determination of immunoreactivity for these options 
was clinically feasible because belonging to a particular group by immune status largely determines the further course of the tumor process and the effectiveness of the surgical treatment of brain tumors [23].

5. In terms of immune responses, we identified the stages of the immune response. As brain tumor progresses, one state of immunoreactivity can transition into another (e.g., from hyperergic to anergic and vice versa), which confirms the dynamic nature of immune responses upon the surgical treatment of brain tumors.

6 . Based on the results of our research, we could recommend possible immunotherapy options depending on the orientation and type of the body's immune response to tumor growth.

With this work, we wanted to study the state and level of immunity in patients with brain tumors, depending on the histological structure of the tumors. We tried to establish the dynamics of immune responses at different times after surgical treatment of brain tumors and to determine the effectiveness of surgical treatment. We believe that such work opens up prospects for the development of methods of targeted immunotherapy.

\section{Conflict of Interest}

There are no conflicts of interest

\section{References}

[1] Kim J, Bal JS (2016) Tumor-Associated Macrophages and neutrophils in tumor microenvironment. Mediatorainflamm. 6058147. Pubmedpmia: 26966341.

[2] Lisyanyi NI (2014) The content of oncogenic viruses in medulloblastomas and gliomas of the brain. Materials of the international scientific-practical conference. Kiev: 34-36.

[3] Lisyanyi NI (1990) The effect of surgery on the immune system upon brain tumors. Clinical surgery 12: 4-6.

[4] Menter DY, Tucker SC, Kopetz S. et al. (2014) Platelets and cancer: a casual or causal relationship: Revisiter. Cancer Metastasis Rev 33 (1): 231-269.

[5] Ohgaki H, Kleihues P (2007) Genetic pathways to primary and secondary glioblastoma. Am J Pathol 170 (5): 1445-1453.

[6] Waziri A (2010) Glioblastoma-derived mechanisms of systemic immunosuppression. Neurosurg Clin N Am 21 (1): 31-42.

[7] Auezova A et al. (2016) Association of preoperative levels of selected blood inflammatory markers with prognosis in Gliomas. Oncotargetsther 11 (9): 6111-6117.
[8] Wang Q. et al. (2017) Tumor evolution of glioma-intrinsic gene expression subtypes associates with immunological changes in the microenvironment. Cancer Cell 32: 42-56 e46.

[9] Woroniecka KI, Rhodin KE, Chongsathidkiet P, Keith KA, Fecci PE. (2018) T-cell dysfunction in glioblastoma: applying a new framework. Clin. Cancer Res. 24: 3792-3802.

[10] Aquino D, Gioppo A, Finocchiaro G, Bruzzone MG, Cuccarini V. (2017) MRI in glioma immunotherapy: evidence, pitfalls, and perspectives. J. Immunol. Res. 5813951.

[11] Wu A et al. (2019) Combination anti-CXCR4 and anti-PD-1 immunotherapy provides survival benefit in glioblastoma through immune cell modulation of tumor microenvironment. J. Neurooncol. 143: 241-249.

[12] Sampson JH, Gunn MD, Fecci PE, Ashley DM (2019) Brain immunology and immunotherapy in brain tumours. Nature Reviews Cancer. 20: 12-25.

[13] Starchenko AA (2001) Clinical neuroimmunology of surgical diseases of the brain. SPb.: Saint-Petersburg Medical Ed., p. 324.

[14] Chernyh ER (2002) Combined immunotherapy in the treatment of malignant brain tumors. Medical immunology 4 (4-5): 583-592.

[15] Brandlein S, Pohle T, Ruoff N, Wozniak E, Muller“Hermelink HK, Vollmers HP (2003) Natural IgM antibodies and immunosurveillance mechanisms against epithelial cancer cells in humans. Cancer Res. 63 (22): 7995-8005.

[16] Xu LW, Chow KK, Lim M, Li G (2014) Current vaccine trials in glioblastoma: a review. Journal of immunology research 796856.

[17] Gorbunov VI (1996) Immunopathology of a traumatic disease. Ulyanovsk, SHU: p. 71.

[18] Selyukova MV (2004) Features of the immune status in patients with meningiomas and gliomas of the cerebral hemispheres Author's thesis, master's dissertation Kirov: p. 24

[19] Dix AR, Brooks WH, Roszman TL, Morford LA (1999) Immune defects observed in patients with primary malignant brain tumors. J Neuroimmunol 100: 216-232.

[20] CA J, PT, MW, MS (2001) Immunobiology 5: The Immune System in Health and Disease. 5 ed., New York: Garland Publishers.

[21] Bacic D, Uravic M, Bacic R, Sutic I, Petrosic N (2011) Augmentation of regulatory $\mathrm{T}$ cells $(\mathrm{CD} 4+\mathrm{CD} 25+\mathrm{Foxp} 3+)$ correlates with tumor stage in patients with colorectal cancer. Collegium antropologicum 35 Suppl 2: 65-68.

[22] Norden AD, Drappatz J, Wen PY (2008) Novel antiangiogenic therapies for malignant gliomas. Lancet neurology 7 (12): 1152-1160.

[23] Rudenko VA (1990) Subpopulations of lymphocytes and their functional activity in brain tumors. Medical business 9: 91-94. 